\title{
Reliability of sampling strategies for measuring dairy cattle welfare on commercial farms
}

\author{
Jennifer M. C. Van Os, ${ }^{\star}$ Christoph Winckler,† Julia Trieb,† Soraia V. Matarazzo,‡ Terry W. Lehenbauer,§ \\ John D. Champagne, $\S$ and Cassandra B. Tucker*1 \\ ${ }^{*}$ Center for Animal Welfare, Department of Animal Science, University of California, Davis 95616 \\ †Department of Sustainable Agricultural Systems, Division of Livestock Sciences, University of Natural Resources and Life Sciences, Vienna, \\ Austria, 1180 \\ ‡Department of Agricultural and Environmental Sciences, Santa Cruz State University, Ilhéus, Brazil, 45662 \\ $\S$ Veterinary Medicine Teaching and Research Center, University of California-Davis, Tulare 93274
}

\section{ABSTRACT}

Our objective was to evaluate how the proportion of high-producing lactating cows sampled on each farm and the selection method affect prevalence estimates for animal-based measures. We assessed the entire high-producing pen (days in milk $<100$; range $=81$ 241 cows) on 10 California farms using measures from the Welfare Quality Protocol for Cattle. Cows were restrained in head locks and visually evaluated for body condition, dirtiness, skin alterations (hair loss, lesions, or swelling), discharge (ocular, nasal, vulvar), diarrhea, and impaired respiration. Lameness was scored upon release. Prevalence was calculated as a percentage of assessed cows. The most common conditions were dirty hindquarters $(33.5 \pm 10.7 \%$, mean \pm standard deviation) and lesions or swelling on the carpal joint (34.4 \pm $7.0 \%)$ and hock $(26.4 \pm 16.7 \%)$. Diarrhea $(8.0 \pm 5.8 \%)$, lameness (moderate $=7.3 \pm 4.7 \%$, severe $=2.2 \pm$ $2.2 \%)$, and neck $(5.8 \pm 12.6 \%)$, flank $(4.5 \pm 5.0 \%)$, or hindquarter alterations $(5.5 \pm 3.9 \%)$ were less common. Very fat cows, vulvar discharge, and impaired respiration were rare $(\leq 1 \%)$ and were excluded from further analysis. Four sampling strategies were used to generate 20 estimates for each animal-based measure. The strategies were (1) selecting every 10th, 5th, 4th, 3rd, 2nd, 2 of 3 , or 3 of 4 cows at the feed bunk (7 estimates/measure); (2) randomly selecting 7 matching proportions of the pen; (3) randomly selecting cows using 3 sample size calculations from the Welfare Quality Protocol; and (4) selecting the first, middle, or final third of cows exiting the milking parlor. Estimates were compared with true values using regression analysis and were considered accurate if they met 3 criteria: the coefficient of

Received July 31, 2017.

Accepted October 17, 2017.

${ }^{1}$ Corresponding author: cbtucker@ucdavis.edu determination was $\geq 0.9$ and the slope and intercept did not differ significantly from 1 and 0 , respectively. All estimates met the slope and intercept criteria, whereas the coefficient of determination increased when more cows were sampled. All estimates were accurate for neck alterations, ocular discharge $(22.2 \pm 27.4 \%)$, and carpal joint hair loss $(14.1 \pm 17.4 \%)$. Selecting a third of the milking order or using the Welfare Quality sample size calculations failed to accurately estimate all measures simultaneously. However, all estimates were accurate when selecting at least 2 of every 3 cows locked at the feed bunk. Using restraint position at the feed bunk did not differ systematically from computer-selecting the same proportion of cows randomly, and the former may be a simpler approach for welfare assessments.

Key words: health, on-farm assessment, sampling, validation

\section{INTRODUCTION}

Welfare describes an animal's state and spans a spectrum from good to poor. Based on this concept, assessments of farm animal welfare increasingly focus on evaluating animal-based measures (e.g., Whay et al., 2003) in addition to aspects of the environment or management. Animal welfare assurance programs not only seek to accurately classify the state of the animals on a given farm, but are also faced with feasibility constraints, namely investment of time and the number of assessors.

A key issue is the appropriate number of animals to sample on a given farm, as this can affect both the accuracy of welfare classification and the costs associated with conducting the assessment. Studies on dairy cattle (Endres et al., 2014) and swine (Mullan et al., 2009) have shown some associations between the true prevalence of an animal-based measure and the sample size needed to accurately estimate it, such that conditions with low prevalence sometimes require sampling 
more animals. This means that accurately estimating low-prevalence conditions may present a feasibility challenge on larger operations, particularly given the continued global trend of farms increasing in size (reviewed by Robbins et al., 2016).

Assessment schemes have attempted to balance accuracy and feasibility in their guidelines for the number of animals to score. Among the Welfare Quality protocols, these sample size recommendations vary. Within the swine protocol (Welfare Quality, 2009b), certain measures call for different sample sizes, but the number of pigs sampled does not increase directly with farm size. For dairy cattle (Welfare Quality, 2009a), a single sample size, based on Cochran (1977), is used for all animal-based health measures on a given farm, and the number of cows sampled grows at a diminishing rate as herd size increases. To our knowledge, no work has directly evaluated the reliability of using these sample size calculations to estimate prevalence across a large selection of the animal-based measures in the Welfare Quality (2009a) protocol for dairy cattle.

A few studies have focused primarily on lameness, an important animal-based measure. Strategies have included comparing sample estimates against a tolerance range relative to the true prevalence (Main et al., 2010; Hoffman et al., 2013) or using sequential sampling based on a technique used in clinical trials (Heath et al., 2016), although the latter study assumed that the sample size calculations in the Welfare Quality (2009a) protocol represented the true prevalence. One study, however, used a more stringent linear regression approach to evaluate the sample size needed to estimate lameness and other select animal-based measures (Endres et al., 2014). Estimates were deemed accurate only if their relationship to the true prevalence had a coefficient of determination $\left(\mathbf{R}^{2}\right) \geq 0.9$ and the slope and intercept did not differ from 1 and 0 , respectively. Our research group has used the same linear regression criteria to identify schedules for sampling cattle behavior through direct observation (Stackhouse-Lawson et al., 2015; Chen et al., 2016; Tresoldi et al., 2016) or using data loggers (Ledgerwood et al., 2010).

In addition to determining how many cows to sample, questions remain about which animals to select. The Welfare Quality (2009a) protocol for dairy cattle provides recommendations such as selecting every $n$th cow either in the milking order or when locked at the feed bunk, or by choosing cattle distributed throughout the home pen and engaged in various activities (e.g., standing, lying, or feeding) to approximate random sampling. In studies that benchmark measures of dairy cattle welfare across farms, a common approach is to score cows during milking (von Keyserlingk et al.,
2012), but a single assessor would be unable to observe the same cow from the front, back, and while walking to score all of the animal-based measures in the Welfare Quality (2009a) assessment.

Our objective was to evaluate how prevalence estimates for a wide range of animal-based measures are affected by the proportion of high-producing cows sampled and the method used to select them. We focused on this population because considerable interest exists in studying confined, high-producing cattle (e.g., Espejo et al., 2006; von Keyserlingk et al., 2012; Cook et al., 2016), which are particularly at risk for welfare concerns such as lameness (Barkema et al., 1994). We evaluated the accuracy of the estimates generated by sampling various proportions of cattle. In addition, we compared the strategies of choosing cows based on their position while restrained at the feed bunk, selecting ear tag numbers randomly, or assessing different cows at separate times during milking.

\section{MATERIALS AND METHODS}

\section{Animals, Housing, and Measures}

Between October and December 2013, 2 assessors visited 10 California dairy farms $(\mathrm{n}=10$ farms, 1,805 cows total), all of which milked cows twice daily. These farms were a convenience sample among clients of the Veterinary Medicine Teaching and Research Center of the University of California-Davis (UC Davis) School of Veterinary Medicine. On each farm, all cows in 1 pen of high-producing cows (DIM <100) were assessed. The housing type, breed managed, herd size, and size of the high-producing pen from each farm are shown in Table 1. Each cow was identified by ear tag number and was evaluated using select measures (Table 2) from the dairy cow section of the Welfare Quality (2009a) protocol. The measures selected were all nonbehavioral ones assessed at the individual animal level. The evaluation was conducted while cows were restrained in head locks in the pen with the exception of lameness, which was scored when cows were released. One assessor recorded ear tag numbers and nasal and ocular discharge from the front of the cow. The second assessor recorded all other measures from inside of the pen, releasing 1 cow at a time from the head locks to score lameness and to gain a full side view of the next cow. Intraobserver reliability was determined using a combination of photos, video clips, and live observations ( $\geq 20$ cows per measure), and percentage agreement was $\geq 80 \%$ for all measures. Cow position along the feed bunk during restraint and the order in which they were milked were also manually recorded. 
Table 1. Characteristics of 10 California dairy farms that were assessed and the number of cows randomly selected in the high-producing pen (DIM <100) using various sample sizes

\begin{tabular}{|c|c|c|c|c|c|c|c|c|c|c|c|c|}
\hline \multirow[b]{2}{*}{ Housing $^{1}$} & \multirow[b]{2}{*}{ Breed } & \multirow[b]{2}{*}{$\begin{array}{l}\text { Pen } \\
\text { size }\end{array}$} & \multicolumn{3}{|c|}{ Equation $^{2}$} & \multicolumn{7}{|c|}{ Proportion of pen ${ }^{3}$} \\
\hline & & & Infinite & $\begin{array}{l}\text { WQ } \\
\text { high }\end{array}$ & $\begin{array}{l}\text { WQ } \\
\text { low }\end{array}$ & $1 / 10$ & $1 / 5$ & $1 / 4$ & $1 / 3$ & $1 / 2$ & $2 / 3$ & $3 / 4$ \\
\hline \multirow[t]{4}{*}{ Freestall } & Holstein-Friesian & 241 & 96 & 69 & 53 & 24 & 48 & 60 & 80 & 121 & 161 & 181 \\
\hline & & 216 & 96 & 66 & 51 & 22 & 43 & 54 & 72 & 108 & 144 & 162 \\
\hline & & 191 & 96 & 64 & 50 & 19 & 38 & 48 & 64 & 96 & 127 & 143 \\
\hline & & 145 & 96 & 57 & 46 & 15 & 29 & 36 & 48 & 73 & 97 & 110 \\
\hline \multirow[t]{4}{*}{ Dry lot } & Holstein-Friesian & 233 & 96 & 68 & 52 & 23 & 47 & 58 & 78 & 117 & 155 & 174 \\
\hline & & 227 & 96 & 67 & 52 & 23 & 45 & 57 & 76 & 114 & 151 & 170 \\
\hline & & 81 & - & 44 & 37 & 8 & 16 & 20 & 27 & 41 & 54 & 61 \\
\hline & Jersey & 204 & 96 & 65 & 51 & 20 & 41 & 51 & 68 & 102 & 136 & 153 \\
\hline
\end{tabular}

${ }^{1}$ Housing types were either freestall barns with sand bedding or dry lot corrals with dirt surfaces.

${ }^{2}$ Sample sizes were based on the equations by Cochran (1977) used to calculate the recommendations in the Welfare Quality (2009a) protocol, all using an assumed prevalence of $50 \%$ for a given health condition and a desired precision of $10 \%$. Infinite refers to a sample size of 96 cows, based on a 95\% CI and an infinite population size, independent of actual pen size. WQ high and WQ low refer to sample sizes based on 95 and $90 \%$ CI, respectively, along with the actual pen sizes for each farm.

${ }^{3}$ Sample sizes reflected proportions of the population in the pen, with cows selected both randomly by computer and based on their positions at the feed bunk.

\section{Methods for Selecting Cows to Sample}

Ear tag numbers were selected using 4 strategies, and the individual assessment data from the selected cows were used to create a total of 20 subsets of data. The first 2 strategies were to select different proportions of the pen (Table 1; 14 subsets of data). First, to reflect how an assessor would potentially identify cows when locked at the feed bunk, 7 subsets of data were created by selecting every 10 th, 5 th, 4th, 3rd, 2 nd, 2 out of every 3 , and 3 out of every 4 cows. Second, we created 7 subsets of data reflecting the same proportions of the pen, generated using the simple random sample function within PROC SURVEYSELECT in SAS software (SAS Institute, 2009).

The third strategy (Table 1; 3 subsets of data) used the equations by Cochran (1977) to determine sample size, where $p=$ the expected prevalence of the condition, $Z=$ the confidence interval, $d=$ the desired precision, and $N=$ the population (pen) size:

$$
\begin{aligned}
& n_{\mathrm{inf}}=\frac{p(1-p) Z^{2}}{d^{2}} \\
& n_{\mathrm{fin}}=\frac{n_{\mathrm{inf}}}{1+\left(n_{\mathrm{inf}}-1\right) / N} .
\end{aligned}
$$

All 3 calculations used $p=50 \%$ and $d=10 \%$. The $n_{\text {inf }}$ calculation for an infinite population, using $Z=1.96$ (95\% CI), generated a sample size of 96 cows; on the 9 farms with sufficient pen size, this number of ear tags was randomly selected using PROC SURVEYSELECT. The other 2 subsets were generated based on the recommendations in the Welfare Quality (2009a) protocol, which determine $n_{\text {fin }}$ using the pen size of each farm and either $Z=1.96$ (WQ high) or 1.645 (90\% CI; WQ low).

The fourth strategy was to divide each pen into 3 equal subsets based on the order in which the cows were milked (first, middle, and final third).

\section{Statistical Analysis}

The data were compiled into 25 measures (19 individual and 6 composite), with prevalence for each calculated as a percentage of the number of cows sampled on each farm. Very fat cows, impaired respiration, and vulvar discharge were rarely observed $(\leq 1 \%$ of cows on any farm; Table 2), and were thus excluded from analysis. For the remaining 22 measures (16 individual, 6 composite), we evaluated relationships between the true prevalence and the estimates generated from each subset using linear regressions (PROC REG; SAS Institute, 2009). A subset of data was determined to accurately reflect the true prevalence if it met 3 criteria: the $\mathrm{R}^{2}$ was $\geq 0.9$, the slope did not differ significantly from $1(P>0.05)$, and the intercept did not differ significantly from $0(P>0.05)$. To control for the false discovery rate associated with conducting multiple comparisons $(\mathrm{m}=880)$, all $P$-values for the slopes and intercepts were adjusted using the Benjamini and Hochberg (1995) procedure in PROC MULTTEST. 


\section{RESULTS AND DISCUSSION}

\section{Prevalence of Health Conditions}

Descriptive data for the true prevalence of each measure are presented in Table 2. To place our results for average prevalence in the context of existing studies, we selected those that assessed only high-producing (or $<120$ DIM), loose-housed cows across multiple farms. For some measures, particularly severe lameness, the mean and maximum prevalence in our study was relatively low, perhaps because we used a convenience sample of producers who had strong relationships with UC Davis veterinarians. Nonetheless, drawing conclusions about mean prevalence among studies is challenging, as estimates can vary due to differences among populations (e.g., region, housing system, and manage- ment factors) or inconsistencies among scoring systems, assessors, and sampling strategies.

Lameness. Total clinical lameness in our study averaged $9.5 \%$, virtually identical to the mean of $9.6 \%$ recently reported in a sample of 184 US farms across various housing types (Adams et al., 2017). In California, the average among freestall operations was previously found to be 3 times higher (30.8\% in von Keyserlingk et al., 2012). Averages in other regions ranged from 13 to $25 \%$ in the Midwest (Espejo et al., 2006; Cook et al., 2016), 15 to $28 \%$ in Canada (von Keyserlingk et al., 2012; Solano et al., 2015; King et al., 2016; Westin et al., 2016), and $55 \%$ in the northeast United States (von Keyserlingk et al., 2012). Severe lameness in our study averaged $2.2 \%$, consistent with the means of 2.2 to $2.7 \%$ reported in several North American studies (Cook et al., 2016; King et al., 2016; Adams et al., 2017), but

Table 2. Definitions, mean prevalence ${ }^{1}, \mathrm{SD}$, and range across California dairy farms $(\mathrm{n}=10)$ for 23 measures from the Welfare Quality (2009a) protocol

\begin{tabular}{|c|c|c|c|c|}
\hline Measure & Definition & Prevalence & $\mathrm{SD}$ & Range \\
\hline Very lean $^{2}$ & $\begin{array}{l}\text { Depressions around tail head and loin; prominent tail head, hip bones, } \\
\text { spine, ribs }\end{array}$ & 13.0 & 10.4 & $1.2-34.3$ \\
\hline Very fat ${ }^{2}$ & $\begin{array}{l}\text { Convex between hip bones and spine, transverse processes } \\
\text { indiscernible, fatty tissue visible under skin }\end{array}$ & 0.2 & 0.4 & $0-1.0$ \\
\hline Any dirtiness ${ }^{2}$ & Dirtiness on any of the 3 assessed body parts & 46.8 & 12.4 & $18.3-62.8$ \\
\hline Lower hind leg & Plaques $^{3}$ of dirt above the coronary band & 18.4 & 15.5 & $2.6-46.9$ \\
\hline Hindquarter & $\begin{array}{l}\text { Plaques of dirt on the upper hind leg, flank, tail, or rear (excluding } \\
\text { udder) }\end{array}$ & 33.5 & 10.7 & $16.8-48.9$ \\
\hline Moderate & Imperfect temporal rhythm in stride creating a limp & 7.3 & 4.7 & $1.4-14.2$ \\
\hline Severe & $\begin{array}{l}\text { More than one affected limb or strong reluctance to bear weight on one } \\
\text { limb }\end{array}$ & 2.2 & 2.2 & $0-5.7$ \\
\hline Nonlimb alterations ${ }^{5}$ & Any hair loss, ${ }^{6}$ lesion, ${ }^{7}$ or swelling & & & \\
\hline Hindquarter & Upper hind leg, tail, or rear & 5.5 & 3.9 & $1.4-14.1$ \\
\hline Flank & Flank, side, or udder & 4.5 & 5.0 & $0-15.7$ \\
\hline Carpal joint alterations ${ }^{5}$ & Outer carpal joint nearest the assessor & 47.3 & 15.8 & $23.5-72.7$ \\
\hline Hair loss & & 14.1 & 17.4 & $0-53.7$ \\
\hline Lesions or swelling & & 34.4 & 17.0 & $13.8-62.6$ \\
\hline Any hair loss & Hair loss on at least 1 assessed body region & 30.6 & 19.9 & $10.3-62.0$ \\
\hline Any lesions or swelling & At least 1 lesion or swelling on any assessed body region & 52.2 & 18.0 & $33.2-84.8$ \\
\hline Nasal discharge & Clearly visible discharge or flow from the nostrils & 19.3 & 14.5 & $0.4-47.5$ \\
\hline Ocular discharge & Clearly visible wet or dry discharge or flow from the eye $\geq 3 \mathrm{~cm}$ long & 22.2 & 27.4 & $0-75.7$ \\
\hline Impaired respiration & Deep and labored or overtly difficult breathing & 0.1 & 0.2 & $0-0.7$ \\
\hline Evidence of diarrhea & $\begin{array}{l}\text { Loose watery manure at least the size of a hand on both sides of the } \\
\text { tail below the tail head }\end{array}$ & 8.0 & 5.8 & $0-18.6$ \\
\hline Vulvar discharge & Purulent effluent from vulva or plaques of pus on underside of tail & 0 & 0 & $0-0$ \\
\hline
\end{tabular}

${ }^{1}$ Percentage of cows in the high-producing pen (DIM <100).

${ }^{2}$ Body condition and dirtiness were assessed from behind and from 1 side of the cow.

${ }^{3}$ Plaques were 3-dimensional layers of dirt equivalent to either the size of a palm or half the surface area of the focal body part.

${ }^{4}$ Lameness was assessed from the side or from behind while cows were walking.

${ }^{5}$ Alterations were assessed from the same side of the cow used to score dirtiness and consisted of any hair loss, lesions, or swelling.

${ }^{6}$ Hair loss referred to undamaged areas of the skin with either missing hair or extensive thinning of the coat (i.e., due to parasites) $\geq 2 \mathrm{~cm}$ in diameter.

${ }^{7}$ Lesions included areas of damaged skin $\geq 2 \mathrm{~cm}$ in diameter in the form of a scab, wound, or dermatitis due to parasites. 
lower than the $3.6 \%$ previously found in California (von Keyserlingk et al., 2012) and the 4.0 to $8.2 \%$ reported across other regions of Canada and the United States (Espejo et al., 2006; von Keyserlingk et al., 2012; Westin et al., 2016).

Skin Alterations. On average, the prevalence of hair loss was $15 \%$ on the hock and $14 \%$ on the carpal joint. These values were consistent with 2 studies reporting means of 10 to $15 \%$ on the hock (Zaffino Heyerhoff et al., 2014; Adams et al., 2017) and $13 \%$ on the carpal joint (Zaffino Heyerhoff et al., 2014). All other North American studies reported much higher averages, ranging from 38 to $76 \%$ for the hock (von Keyserlingk et al., 2012; Endres et al., 2014; Cook et al., 2016) and 47\% for the carpal joint (Cook et al., 2016). However, severe alterations (lesions or swelling) were more prevalent in our study, averaging $26 \%$ on the hock and $34 \%$ on the carpal joint. In contrast, most North American studies found means of only 2 to $12 \%$ for severe hock injuries (von Keyserlingk et al., 2012; Endres et al., 2014; Cook et al., 2016; Adams et al., 2017), with the exception of one in Canada reporting 47\% (Zaffino Heyerhoff et al., 2014). Likewise, although studies in Canada and the northeast United States reported means of 23 to $24 \%$ for severe carpal joint injuries (von Keyserlingk et al., 2012; Zaffino Heyerhoff et al., 2014), the averages were only 0.3 to $6 \%$ in California and Wisconsin (von Keyserlingk et al., 2012; Cook et al., 2016). Total neck alterations in our study averaged $5.8 \%$, lower than the $8.6 \%$ previously reported in Wisconsin (Cook et al., 2016) and the $16 \%$ found in Canada (Zaffino Heyerhoff et al., 2014).

Other Conditions. Previous research found that, across Minnesota farms, only $0.1 \%$ of cows had either very high or low body condition (Endres et al., 2014). Similarly, only $0.2 \%$ of cows in our study were very fat; however, we considered a much higher proportion (13\%, on average) of cows to be very lean. This difference could perhaps be explained, in part, by the timing of our assessments in autumn, as body condition can decrease as a result of heat stress (Shwartz et al., 2009). Despite different methods for assessing hygiene, the $46.8 \%$ average prevalence for any dirtiness on the lower hind leg, udder, or hindquarters was similar to the mean of $54.9 \%$ reported for dirtiness on the udder or lower hind legs on farms in Minnesota (Endres et al., 2014).

\section{Methods for Selecting Cows to Sample}

The intercept was not significantly different from 0 $(P \geq 0.31)$ and the slope was not significantly different from $1(P>0.18)$ in any comparison, suggesting a lack of consistent over- or under-estimation across the 10 farms in our study.

Selecting Proportions of the Pen. When a larger proportion of the pen was sampled, the number of accurately estimated measures increased (Table 3; inaccurate estimates are shown in Supplemental Table S1, https://doi.org/10.3168/jds.2017-13611). Selecting at least 2 of every 3 cows accurately captured the prevalence of all measures when choosing cows locked at the feed bunk and accurately estimated all but severe lameness when randomly computer-selecting ear tag numbers. The association between sample size and accuracy was driven by the increase in $\mathrm{R}^{2}$ values when more cows were sampled (2 examples of measures with mean and range prevalence values that were relatively lower and higher, respectively, are shown in Figure 1; all relationships are shown in Supplemental Figure S1, https://doi.org/10.3168/jds.2017-13611). Endres et al. (2014) observed a similar pattern when assessing a narrower range of measures. Selecting an $\mathrm{R}^{2}$ threshold is subjective, and some researchers have used lower cutoffs. For example, Miller-Cushon and DeVries (2011) considered $\mathrm{R}^{2}>0.8$ to indicate a strong association and $\mathrm{R}^{2}>0.7$ to represent a fairly good association because, in their data set, all relationships with $\mathrm{R}^{2}>0.75$ also met the slope and intercept criteria. Based on the same rationale, Madruga et al. (2017) used a threshold of $\mathrm{R}^{2}$ $>0.76$. In the current study, however, even relationships with low $\mathrm{R}^{2}$ values met the slope and intercept criteria. Therefore, we used a conservative threshold of $\mathrm{R}^{2} \geq 0.9$, which our research group also used in other studies to identify sampling schedules for capturing cattle behavior through observation (Stackhouse-Lawson et al., 2015; Chen et al., 2016; Tresoldi et al., 2016) or with data loggers (Ledgerwood et al., 2010).

Both within and across studies, the proportion of cows to sample appears to depend, in part, on the true prevalence of a given condition. In our study, almost all of the measures with $<10 \%$ average prevalence, such as moderate and severe lameness, diarrhea, and flank or hindquarter alterations, required more cows to generate accurate estimates. When Endres et al. (2014) evaluated 52 Minnesota farms, they needed to sample only 15 or $30 \%$ of the high-producing pen to estimate total and severe lameness (24.4 and 6.0\% mean prevalence), respectively. In contrast, the mean lameness prevalence in our population was much lower (9.5\% total, $2.2 \%$ severe), and we needed to sample at least every third or every other cow at the feed bunk, respectively. Likewise, Endres et al. (2014) needed to sample $30 \%$ of the pen to estimate hock lesions or swelling (10.6\% mean prevalence), whereas we could estimate this condition (26.4\% mean prevalence) by selecting every tenth cow 
VAN OS ET AL.

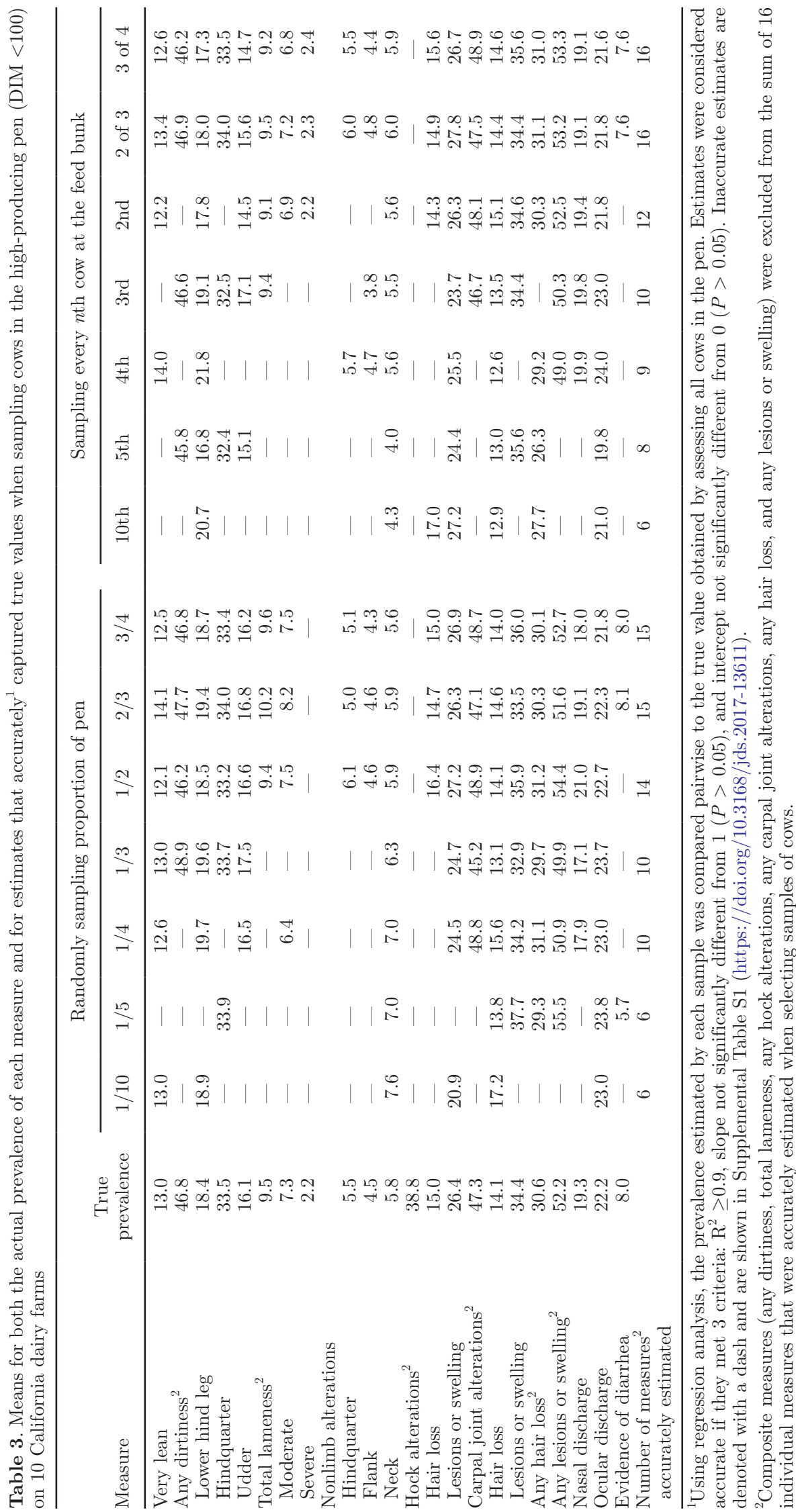


at the feed bunk. Drawing general conclusions about the appropriate proportion of cows to sample is challenging, not only because true prevalence varies across measures and populations, but also because the relationship between true prevalence and how many cows to sample is imperfect. For example, neck alterations were accurately estimated using only $10 \%$ of the pen despite an average prevalence of only $5.8 \%$, and the reasons for this are unclear.

In addition to evaluating the proportion of the pen to sample, we also compared approaches for choosing

\section{(A) Moderate lameness}

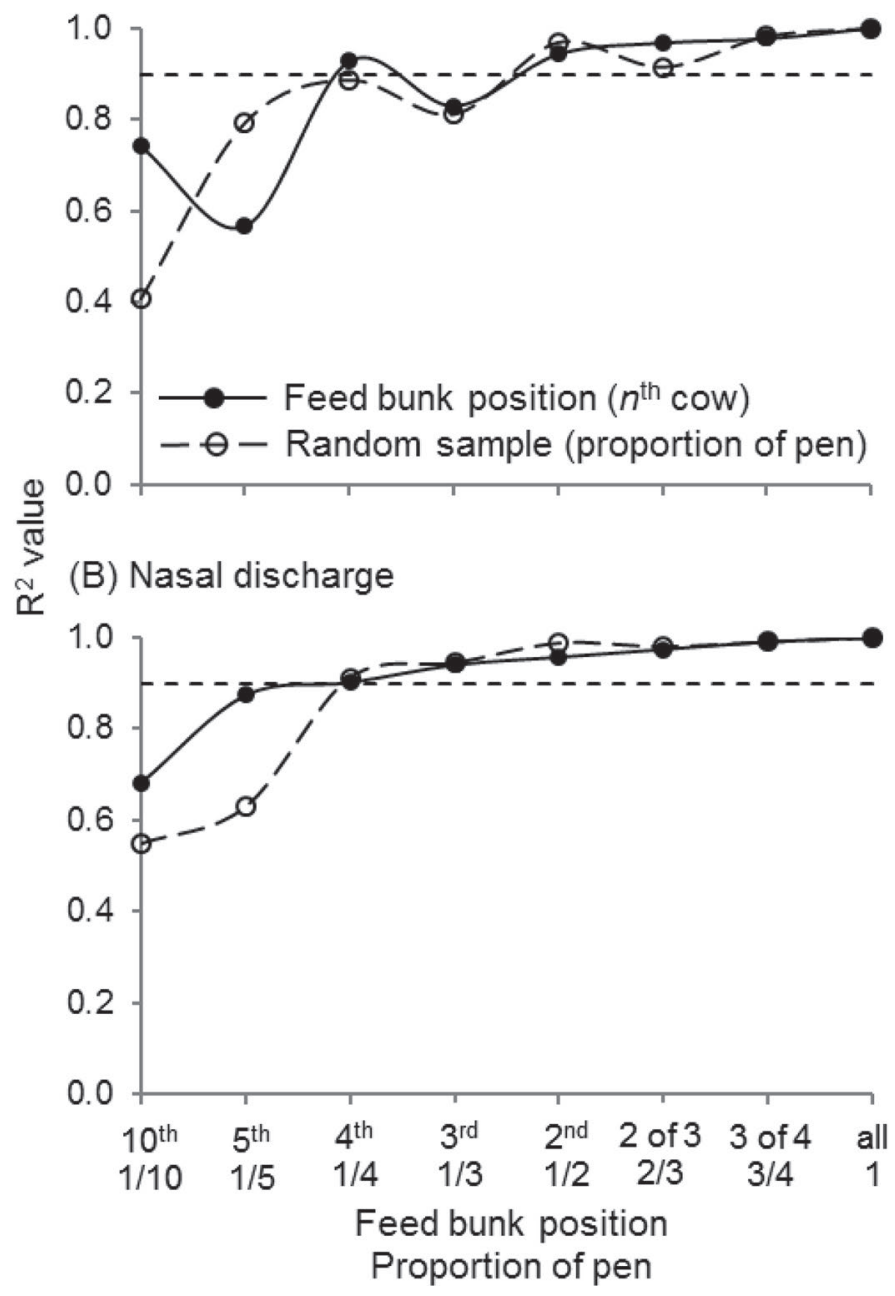

Figure 1. The coefficient of determination $\left(\mathrm{R}^{2}\right)$ for the relationship between the true mean prevalence across 10 farms for (A) moderate lameness and (B) nasal discharge, and the prevalence estimated by sampling a subset of cows as a function of the proportion of the pen sampled. Two methods were used to determine which cows to sample: their position when locked in head gates at the feed bunk (every $n$th cow) or random selection of ear tag numbers. When $\mathrm{R}^{2} \geq 0.9$ (dashed line), this was considered a strong relationship between the true and estimated prevalence. Relationships for the other measures that were assessed are shown in Supplemental Figure S1 (https://doi. org/10.3168/jds.2017-13611). cows. To approximate selecting cows randomly, the Welfare Quality (2009a) protocol suggests identifying those engaging in different activities throughout the pen. However, a potential drawback to this approach is bias resulting from cows being selected because morbidity may make them more conspicuous. In addition, when cows are not restrained, it could be more difficult to score certain measures. An alternative is to randomly preselect ear tag numbers, but identifying specific cattle would be time consuming. A simpler solution would be to assess every $n$th cow while restrained at the feed bunk, and we found this approach did not differ systematically from randomly computer-selecting ear tag numbers.

Sample Sizes Based on Welfare Quality. All 3 equations accurately estimated ocular discharge, neck or flank alterations, hair loss on the carpal joint, lesions or swelling on the hock, and dirtiness on the lower hind leg (Table 4). Randomly selecting 96 cows (40 to $67 \%$ of the pen on a subset of 9 farms) generated accurate estimates for 8 additional measures, but both the WQ high (44-69 cows, $29-54 \%$ of the pen) and WQ low (37-53 cows, $22-46 \%$ of the pen) equations accurately estimated only 2 additional measures. Prevalence for hair loss on the hock and moderate lameness could not be accurately estimated by any of the equations (inaccurate estimates are shown in Supplemental Table S2, https://doi.org/10.3168/jds.2017-13611), and both WQ high and WQ low failed to accurately capture severe lameness or dirtiness on the hindquarters and udder.

The sampling recommendations in the Welfare Quality (2009a) protocol are based on equations (Cochran, 1977) using an expected prevalence of $p=50 \%$, a desired precision of $d=10 \%$, and confidence levels of either 90 or $95 \%$ (WQ low and high, respectively). Seemingly counter to the idea that conditions with lower prevalence may require sampling more cows, the sample size is maximized using $p=50 \%$ because it is multiplied by $1-p$. Compared with selecting the same proportion of the pen across all farms, conducting assessments on large operations may be more practical using the WQ high and low equations because cows are added to the sample at a diminishing rate as the pen size increases. Increasing practicality involves a tradeoff with precision, however, because the number of cows to sample varies inversely with $d^{2}$. The use of $d=10 \%$ may explain why these equations underestimated the number of cows needed to accurately estimate prevalence for many measures in our study. Nonetheless, using $d=5 \%$, as suggested by Naing et al. (2006), may be impractical, as this would increase $n_{\text {inf }}$ by 4 fold and the number of cows in our sample by 1.5 to 2.4 fold. More work is needed to further examine the reliability of the WQ high and low equations across a larger sample with 
more variable pen sizes, as well as to evaluate how farm classifications may be affected when conducting the full Welfare Quality (2009a) assessment.

Milking Order. A total of 12 measures could be accurately estimated by sampling thirds of the milking order (Table 5; inaccurate estimates are shown in Supplemental Table S3, https://doi.org/10.3168/jds .2017-13611) compared with 11 when selecting 1 in 3 cows using feed bunk position or randomly. Within a given third of the milking order, a maximum of 7 to 9 measures were accurately estimated, and these always included ocular discharge, neck alterations, carpal joint hair loss, and lesions or swelling on the hock. In contrast, the prevalence of moderate and total lameness, hindquarter alterations or dirtiness, and signs of diarrhea could not be estimated from any third of the milking order.

Both severe lameness and very lean cows could be estimated accurately using the middle third of the milking order, but these were under- and over-estimated by sampling from the first or final third, respectively. Indeed, in the final third of the milking order, both moderate and severe lameness had double the prevalence compared with the earlier thirds. Others have similarly found more lame cows (Walker et al., 2008; Main et al., 2010) later in the milking order. Although previous researchers found that total lameness could be estimated within $5 \%$ of the true prevalence when sampling from the middle of the milking order (Main et al., 2010; Hoffman et al., 2013), those findings have not been replicated using a statistical comparison with farm as the experimental unit. In our study, moderate and total lameness could not be estimated using any third of the milking order.

If the goal is to accurately capture all measures, reliable prevalence estimates could not be obtained by sampling cows from separate thirds of the milking order. However, a welfare assessment focusing on only a few measures could feasibly score cows from different thirds while they are in the parlor. Hock alterations

Table 4. Means for both the true prevalence of each measure and for estimates that accurately ${ }^{1}$ captured true values when randomly sampling ${ }^{2}$ cows in the high-producing pen $($ DIM $<100)$ on California dairy farms

\begin{tabular}{|c|c|c|c|c|}
\hline Measure & True & Infinite & WQ high & WQ low \\
\hline Very lean & 13.0 & 13.8 & 13.7 & - \\
\hline Any dirtiness ${ }^{3}$ & 46.8 & 46.6 & 45.9 & - \\
\hline Lower hind leg & 18.4 & 20.0 & 16.8 & 16.9 \\
\hline Hindquarter & 33.5 & 31.9 & - & - \\
\hline Udder & 16.1 & 17.4 & - & - \\
\hline Total lameness $^{3}$ & 9.5 & 11.5 & - & - \\
\hline Moderate & 7.3 & - & - & - \\
\hline Severe & 2.2 & 2.3 & - & - \\
\hline \multicolumn{5}{|l|}{ Nonlimb alterations } \\
\hline Hindquarter & 5.5 & 5.8 & - & - \\
\hline Flank & 4.5 & 4.7 & 4.7 & 5.4 \\
\hline Neck & 5.8 & 6.1 & 6.4 & 5.5 \\
\hline Hock alterations ${ }^{3}$ & 38.8 & - & - & - \\
\hline Hair loss & 15.0 & - & - & - \\
\hline Lesions or swelling & 26.4 & 24.8 & 27.4 & 26.2 \\
\hline Carpal joint alterations $^{3}$ & 47.3 & 47.5 & - & 45.8 \\
\hline Hair loss & 14.1 & 13.3 & 13.8 & 13.1 \\
\hline Lesions or swelling & 34.4 & 35.1 & - & 33.6 \\
\hline Any hair $\operatorname{loss}^{3}$ & 30.6 & 31.6 & 29.2 & 28.2 \\
\hline Any lesions or swelling ${ }^{3}$ & 52.2 & 51.2 & 54.0 & 51.7 \\
\hline Nasal discharge & 19.3 & 20.7 & - & 16.5 \\
\hline Ocular discharge & 22.2 & 24.5 & 20.4 & 22.5 \\
\hline Evidence of diarrhea & 8.0 & 9.0 & 7.6 & - \\
\hline Number of measures ${ }^{3}$ accurately estimated & & 14 & 8 & 8 \\
\hline
\end{tabular}

${ }^{1}$ Using regression analysis, the prevalence estimated by each random sample was compared pairwise to the true value obtained by assessing all cows in the pen. Estimates were considered accurate if they met 3 criteria: $\mathrm{R}^{2}$ $\geq 0.9$, slope not significantly different from $1(P>0.05)$, and intercept not significantly different from $0(P$ $>0.05$ ). Inaccurate estimates are denoted with a dash and are shown in Supplemental Table S3 (https://doi. org/10.3168/jds.2017-13611).

${ }^{2}$ Sample sizes were based on the equations used to calculate the Welfare Quality (2009a) recommendations, all using an assumed prevalence of $50 \%$ for a given health condition and a desired precision of $10 \%$. Infinite refers to a sample of 96 cows, based on a $95 \%$ CI and an infinite population size, independent of actual pen size (subset of 9 farms with $\geq 96$ cows/pen). WQ high and WQ low refer to sample sizes based on 95 and $90 \%$ CI, respectively, along with the actual pen sizes on each of the 10 farms.

${ }^{3}$ Composite measures (any dirtiness, total lameness, any hock alterations, any carpal joint alterations, any hair loss, and any lesions or swelling) were excluded from the sum of 16 individual measures that were accurately estimated when sampling cows randomly. 
Table 5. Means for both the true prevalence of each measure and for estimates that accurately ${ }^{1}$ captured true values when sampling the first, middle, or last third of cows in the milking order on 10 California dairy farms

\begin{tabular}{|c|c|c|c|c|}
\hline \multirow[b]{2}{*}{ Measure } & \multirow{2}{*}{$\begin{array}{c}\text { True } \\
\text { prevalence }\end{array}$} & \multicolumn{3}{|c|}{ Third of milking order } \\
\hline & & First & Middle & Last \\
\hline Very lean & 13.0 & - & 13.9 & - \\
\hline Any dirtiness ${ }^{2}$ & 46.8 & - & - & - \\
\hline Lower hind leg & 18.4 & - & 17.1 & 16.2 \\
\hline Hindquarter & 33.5 & - & - & - \\
\hline Udder & 16.1 & 17.9 & - & 15.0 \\
\hline Total lameness $^{2}$ & 9.5 & - & - & - \\
\hline Moderate & 7.3 & - & - & - \\
\hline Severe & 2.2 & - & 1.5 & - \\
\hline \multicolumn{5}{|l|}{ Nonlimb alterations } \\
\hline Hindquarter & 5.5 & - & - & - \\
\hline Flank & 4.5 & 4.7 & - & 4.9 \\
\hline Neck & 5.8 & 5.5 & 6.9 & 5.2 \\
\hline Hock alterations $^{2}$ & 38.8 & - & 38.4 & - \\
\hline Hair loss & 15.0 & 13.7 & - & - \\
\hline Lesions or swelling & 26.4 & 24.2 & 25.1 & 29.5 \\
\hline Carpal joint alterations $^{2}$ & 47.3 & 48.1 & 45.9 & - \\
\hline Hair loss & 14.1 & 14.1 & 12.8 & 15.6 \\
\hline Lesions or swelling & 34.4 & 35.2 & 34.3 & - \\
\hline Any hair loss ${ }^{2}$ & 30.6 & 30.6 & 30.6 & 30.7 \\
\hline Any lesions or swelling ${ }^{2}$ & 52.2 & 51.2 & 49.3 & - \\
\hline Nasal discharge & 19.3 & 20.7 & 17.6 & - \\
\hline Ocular discharge & 22.2 & 22.1 & 22.5 & 21.9 \\
\hline Evidence of diarrhea & 8.0 & - & - & - \\
\hline Number of measures ${ }^{2}$ accurately estimated & & 9 & 9 & 7 \\
\hline
\end{tabular}

${ }^{1}$ Using regression analysis, the prevalence estimated by each sample was compared pairwise to the true value obtained by assessing all cows in the pen. Estimates were considered accurate if they met 3 criteria: $\mathrm{R}^{2} \geq 0.9$, slope not significantly different from $1(P>0.05)$, and intercept not significantly different from $0(P>$ 0.05). Inaccurate estimates are denoted with a dash and are shown in Supplemental Table S3 (https://doi. org/10.3168/jds.2017-13611).

${ }^{2}$ Composite measures (any dirtiness, total lameness, any hock alterations, any carpal joint alterations, any hair loss, and any lesions or swelling) were excluded from the sum of 16 individual measures that were accurately estimated when sampling cows from each third of the milking order.

must be scored from behind during the first third of milking for accuracy, but other measures offered some flexibility in when they could be collected. While scoring hock alterations at the beginning of milking, udder dirtiness and flank alterations could be concurrently collected. The latter measures could also be recorded during the final third of milking, at which time lower hind leg dirtiness could be added. All measures that required observing the front of the cow could be accurately estimated during either the first or middle thirds of milking. A topic for future study could be whether assessing cows in the parlor is more time consuming than doing so in the pen due to wait time during milking.

\section{CONCLUSIONS}

As the proportion of cows sampled increased, so did the $R^{2}$ value for the relationship between estimates and true prevalence, resulting in greater accuracy. In general, many low-prevalence measures required sampling more cows to generate accurate estimates. Reli- able prevalence estimates could not be obtained for all measures by sampling cows from separate thirds of the milking order or when using the sample size calculations in the Welfare Quality (2009a) protocol. In contrast, all estimates were accurate when selecting at least 2 of every 3 cows restrained at the feed bunk. Choosing cows based on their position when restrained at the feed bunk did not differ systematically from randomly computer-selecting ear tags, and the former may be a simpler approach for on-farm welfare assessments.

\section{ACKNOWLEDGMENTS}

The authors express appreciation to the farms that participated in this study, Thomas Graham (Veterinarians Without Borders, Davis, CA) for assistance with identifying participants, and the USDA multistate research project NC1029 (Washington, DC). We gratefully acknowledge the Marshall Plan Scholarship Foundation (Vienna, Austria; funding for J. Trieb) and the infrastructure support of the Department of Animal Science, College of Agricultural and Environmental 
Sciences, and the UC Davis California Agricultural Experiment Station.

\section{REFERENCES}

Adams, A. E., J. E. Lombard, C. P. Fossler, I. N. Román-Muñiz, and C. A. Kopral. 2017. Associations between housing and management practices and the prevalence of lameness, hock lesions, and thin cows on US dairy operations. J. Dairy Sci. 100:2119-2136. https://doi.org/10.3168/jds.2016-11517.

Barkema, H. W., J. D. Westrik, K. A. S. van Keulen, Y. H. Schukken, and A. Brand. 1994. The effects of lameness on reproductive performance, milk production and culling in Dutch dairy farms. Prev. Vet. Med. 20:249-259. https://doi.org/10.1016/0167 $-5877(94) 90058-2$

Benjamini, Y., and Y. Hochberg. 1995. Controlling the false discovery rate: A practical and powerful approach to multiple testing. J. R. Stat. Soc. B (Methodol.) 57:289-300.

Chen, J. M., K. E. Schütz, and C. B. Tucker. 2016. Technical note Comparison of instantaneous sampling and continuous observation of dairy cattle behavior in freestall housing. J. Dairy Sci. 99:8341-8346. https://doi.org/10.3168/jds.2016-11351.

Cochran, W. G. 1977. Sampling Techniques. 3rd ed. Wiley \& Sons, New York, NY.

Cook, N. B., J. P. Hess, M. R. Foy, T. B. Bennett, and R. L. Brotzman. 2016. Management characteristics, lameness, and body injuries of dairy cattle housed in high-performance dairy herds in Wisconsin. J. Dairy Sci. 99:5879-5891. https://doi.org/10.3168/jds.2016 -10956 .

Endres, M. I., K. M. Lobeck-Luchterhand, L. A. Espejo, and C. B. Tucker. 2014. Evaluation of the sample needed to accurately estimate outcome-based measurements of dairy welfare on farm. J. Dairy Sci. 97:3523-3530. https://doi.org/10.3168/jds.2013-7464.

Espejo, L. A., M. I. Endres, and J. A. Salfer. 2006. Prevalence of lameness in high-producing Holstein cows housed in freestall barns in Minnesota. J. Dairy Sci. 89:3052-3058. https://doi.org/10.3168/ jds.S0022-0302(06)72579-6.

Heath, C. A. E., D. C. J. Main, S. Mullan, M. J. Haskell, and W J. Browne. 2016. Sequential sampling: A novel method in farm animal welfare assessment. Animal 10:349-356. https://doi.org/10 $.1017 /$ S1751731115001536.

Hoffman, A. C., D. A. Moore, J. R. Wenz, and J. Vanegas. 2013 Comparison of modeled sampling strategies for estimation of dairy herd lameness prevalence and cow-level variables associated with lameness. J. Dairy Sci. 96:5746-5755. https://doi.org/10.3168/jds $.2013-6891$

King, M. T. M., E. A. Pajor, S. J. LeBlanc, and T. J. DeVries. 2016 Associations of herd-level housing, management, and lameness prevalence with productivity and cow behavior in herds with automated milking systems. J. Dairy Sci. 99:9069-9079. https://doi .org/10.3168/jds.2016-11329.

Ledgerwood, D. N., C. Winckler, and C. B. Tucker. 2010. Evaluation of data loggers, sampling intervals, and editing techniques for measuring the lying behavior of dairy cattle. J. Dairy Sci. 93:51295139. https://doi.org/10.3168/jds.2009-2945.

Madruga, A., E. Mainau, L. A. Gonzalez, M. Rodrigues-Prado, J. L. Ruiz de la Torre, X. Manteca, and A. Ferret. 2017. Technical note: Recording rules for behavioral studies in growing heifers fed highconcentrate diets. J. Anim. Sci. 95:2339-2344. https://doi.org/10 $.2527 /$ jas.2016.1037.

Main, D. C. J., Z. E. Barker, K. A. Leach, N. J. Bell, H. R. Whay, and W. J. Browne. 2010. Sampling strategies for monitoring lameness in dairy cattle. J. Dairy Sci. 93:1970-1978. https://doi.org/10 $.3168 /$ jds.2009-2500.
Miller-Cushon, E. K., and T. J. DeVries. 2011. Technical note: Validation of methodology for characterization of feeding behavior in dairy calves. J. Dairy Sci. 94:6103-6110. https://doi.org/10.3168/ jds.2011-4589.

Mullan, S., W. J. Browne, S. A. Edwards, A. Butterworth, H. R. Whay, and D. C. J. Main. 2009. The effect of sampling strategy on the estimated prevalence of welfare outcome measures on finishing pig farms. Appl. Anim. Behav. Sci. 119:39-48. https://doi.org/10 .1016/j.applanim.2009.03.008.

Naing, L., T. Winn, and B. N. Rusli. 2006. Practical issues in calculating the sample size for prevalence studies. Arch. Orofac. Sci. $1: 9-14$

Robbins, J. A., M. A. G. von Keyserlingk, D. Fraser, and D. M. Weary. 2016. Invited review: Farm size and animal welfare. J. Anim. Sci. 94:5439-5455. https://doi.org/10.2527/jas.2016-0805.

SAS Institute. 2009. User's Guide: Statistics, Version 9.2 ed. SAS Institute, Inc., Cary, NC.

Shwartz, G., M. L. Rhoads, M. J. VanBaale, R. P. Rhoads, and L. H. Baumgard. 2009. Effects of a supplemental yeast culture on heatstressed lactating Holstein cows. J. Dairy Sci. 92:935-942. https:// doi.org/10.3168/jds.2008-1496.

Solano, L., H. W. Barkema, E. A. Pajor, S. Mason, S. J. LeBlanc, J. C. Zaffino Heyerhoff, C. G. R. Nash, D. B. Haley, E. Vasseur, D. Pellerin, J. Rushen, A. M. de Passillé, and K. Orsel. 2015. Prevalence of lameness and associated risk factors in Canadian HolsteinFriesian cows housed in freestall barns. J. Dairy Sci. 98:6978-6991. https://doi.org/10.3168/jds.2015-9652.

Stackhouse-Lawson, K. R., C. B. Tucker, M. S. Calvo-Lorenzo, and F. M. Mitloehner. 2015. Effects of growth-promoting technology on feedlot cattle behavior in the 21 days before slaughter. Appl. Anim. Behav. Sci. 162:1-8. https://doi.org/10.1016/j.applanim .2014.11.001.

Tresoldi, G., K. E. Schütz, and C. B. Tucker. 2016. Assessing heat load in drylot dairy cattle: Refining on-farm sampling methodology. J. Dairy Sci. 99:8970-8980. https://doi.org/10.3168/jds.2016-11353.

von Keyserlingk, M. A. G., A. Barrientos, K. Ito, E. Galo, and D M. Weary. 2012. Benchmarking cow comfort on North American freestall dairies: Lameness, leg injuries, lying time, facility design, and management for high-producing Holstein dairy cows. J. Dairy Sci. 95:7399-7408. https://doi.org/10.3168/jds.2012-5807.

Walker, S. L., R. F. Smith, J. E. Routly, D. N. Jones, M. J. Morris, and H. Dobson. 2008. Lameness, activity time-budgets, and estrus expression in dairy cattle. J. Dairy Sci. 91:4552-4559. https://doi .org/10.3168/jds.2008-1048.

Welfare Quality. 2009a. Welfare Quality Assessment Protocol for Cattle. Welfare Quality Consortium, Lelystad, the Netherlands.

Welfare Quality. 2009b. Welfare Quality Assessment Protocol for Pigs. Welfare Quality Consortium, Lelystad, the Netherlands.

Westin, R., A. Vaughan, A. M. de Passillé, T. J. DeVries, E. A. Pajor, D. Pellerin, J. M. Siegford, A. Witaifi, E. Vasseur, and J. Rushen. 2016. Cow- and farm-level risk factors for lameness on dairy farms with automated milking systems. J. Dairy Sci. 99:3732-3743. https://doi.org/10.3168/jds.2015-10414.

Whay, H. R., D. C. J. Main, L. E. Green, and A. J. F. Webster. 2003 Assessment of the welfare of dairy cattle using animal-based measurements: Direct observations and investigation of farm records. Vet. Rec. 153:197-202

Zaffino Heyerhoff, J. C., S. J. LeBlanc, T. J. DeVries, C. G. R. Nash, J. Gibbons, K. Orsel, H. W. Barkema, L. Solano, J. Rushen, A. M. de Passillé, and D. B. Haley. 2014. Prevalence of and factors associated with hock, knee, and neck injuries on dairy cows in freestall housing in Canada. J. Dairy Sci. 97:173-184. https://doi .org/10.3168/jds.2012-6367. 